www.nature.com $/ \mathrm{hr}$

\title{
Effects of continuous vs. interval exercise training on blood pressure and arterial stiffness in treated hypertension
}

\author{
Guilherme Veiga Guimarães ${ }^{1,2}$, Emmanuel Gomes Ciolac ${ }^{1,3}$, Vitor Oliveira Carvalho ${ }^{1}$, \\ Veridiana Moraes D'Avila ${ }^{1,2}$, Luiz Aparecido Bortolotto ${ }^{1}$ and Edimar Alcides Bocchi ${ }^{1}$
}

Exercise is an effective intervention for treating hypertension and arterial stiffness, but little is known about which exercise modality is the most effective in reducing arterial stiffness and blood pressure in hypertensive subjects. Our purpose was to evaluate the effect of continuous vs. interval exercise training on arterial stiffness and blood pressure in hypertensive patients. Sixty-five patients with hypertension were randomized to 16 weeks of continuous exercise training $(n=26)$, interval training $(n=26)$ or a sedentary routine $(n=13)$. The training was conducted in two $40-\mathrm{min}$ sessions a week. Assessment of arterial stiffness by carotid-femoral pulse wave velocity (PWV) measurement and 24-h ambulatory blood pressure monitoring (ABPM) were performed before and after the 16 weeks of training. At the end of the study, ABPM blood pressure had declined significantly only in the subjects with higher basal values and was independent of training modality. PWV had declined significantly only after interval training from $9.44 \pm 0.91$ to $8.90 \pm 0.96 \mathrm{~m} \mathrm{~s}^{-1}, P=0.009$ (continuous from $10.15 \pm 1.66$ to $9.98 \pm 1.81 \mathrm{~m} \mathrm{~s}^{-1}, P=\mathrm{ns}$; control from $10.23 \pm 1.82$ to $\left.10.53 \pm 1.97 \mathrm{~m} \mathrm{~s}^{-1}, P=\mathrm{ns}\right)$. Continuous and interval exercise training were beneficial for blood pressure control, but only interval training reduced arterial stiffness in treated hypertensive subjects. Hypertension Research (2010) 33, 627-632; doi:10.1038/hr.2010.42; published online 9 April 2010

Keywords: arterial stiffness; blood pressure; exercise

\section{INTRODUCTION}

A sedentary lifestyle has been characterized as an independent risk factor for cardiovascular disease. ${ }^{1}$ Studies have shown the inverse association between physical activity level and the incidence of cardiovascular diseases. ${ }^{2,3,4}$ Various mechanisms could be involved in the cardiovascular protective effects of physical activity, including improvement in endothelial function, a decrease in sympathetic neural activity and a reduction in arterial stiffness. Increased arterial stiffness has been associated with hypertension in elderly subjects ${ }^{5,6}$ and with cardiovascular risk in middle-aged hypertensive patients. Several studies have shown the beneficial effects of exercise on arterial stiffness in different diseases. However, the best exercise modality for better control of hypertension and improved arterial function has not been well established.

Continuous exercise training is the type of physical activity most frequently recommended to hypertensive subjects. ${ }^{7}$ However, some studies have shown that interval exercise training may have greater effects on exercise capacity in healthy subjects ${ }^{8,9}$ and those with cardiovascular disorders. ${ }^{10}$ Interval training promotes greater gradients of shear stress because patients fluctuate between high and low intensities. ${ }^{11}$ We have recently shown that interval exercise acutely decreases blood pressure in hypertensive patients. ${ }^{12}$ We therefore hypothesized that interval exercise has a better effect on arterial stiffness and blood pressure control than continuous exercise does in hypertensive subjects.

The aim of this study was to compare the impact on arterial stiffness of a continuous exercise protocol with one involving interval exercise training, as evaluated by pulse wave velocity (PWV) measurement and 24-h blood pressure control in treated hypertensive subjects.

\section{METHODS}

Study population

We studied 65 sedentary hypertensive subjects ( 23 male, 42 female) on antihypertensive medication and controlled office blood pressure (systolic blood pressure $<140 \mathrm{~mm} \mathrm{Hg}$ and diastolic blood pressure $<90 \mathrm{~mm} \mathrm{Hg}$ on antihypertensive drugs) without complications for at least 3 months. Subjects were recruited from the hypertension department of a tertiary hospital and randomized into three groups: continuous, interval and control. The characteristics of the population are listed in Table 1. We excluded subjects with diagnosed coronary artery disease or an abnormal electrocardiogram during the exercise

${ }^{1}$ Faculdade de Medicina, Heart Institute (InCor), Hospital das Clínicas, Universidade de São Paulo, São Paulo, Brazil; ${ }^{2}$ Laboratory of Physical Activity and Health (LAtiS), Centro de Práticas Esportivas, Universidade de São Paulo, São Paulo, Brazil and ${ }^{3}$ Laboratory of Kinesiology, Instituto de Ortopedia e Traumatologia, Hospital das Clínicas, Faculdade de Medicina, Universidade de São Paulo, São Paulo, Brazil

Correspondence: Dr GV Guimarães, Faculdade de Medicina, Heart Institute, Universidade de São Paulo, Rua Baeta Neves, 98 Sao Paulo, CEP 05444-050, Brazil.

E-mail: gvguima@usp.br

Received 3 November 2009; revised 10 February 2010; accepted 14 February 2010; published online 9 April 2010 
Table 1 Subjects' characteristics

\begin{tabular}{|c|c|c|c|}
\hline & Continuous & Interval & Control \\
\hline Number of subjects & 16 & 16 & 11 \\
\hline $\operatorname{Sex}(F / M)$ & $9 / 7$ & $12 / 4$ & $9 / 2$ \\
\hline Age (years) & $50 \pm 8$ & $45 \pm 9$ & $47 \pm 6$ \\
\hline $\mathrm{BMI}\left(\mathrm{kg} \mathrm{m}^{-2}\right)$ & $28 \pm 4$ & $29 \pm 5$ & $26 \pm 5$ \\
\hline Waist $(\mathrm{cm})$ & $92 \pm 12$ & $91 \pm 10$ & $84 \pm 11$ \\
\hline W/H & $0.91 \pm 0.07$ & $0.88 \pm 0.07$ & $0.87 \pm 0.06$ \\
\hline $\mathrm{PWV}\left(\mathrm{ms}^{-1}\right)$ & $10.15 \pm 1.66$ & $9.44 \pm 0.91$ & $10.23 \pm 1.82$ \\
\hline \multicolumn{4}{|l|}{ Blood pressure } \\
\hline Systolic & $124 \pm 9$ & $125 \pm 9$ & $128 \pm 9$ \\
\hline Diastolic & $81 \pm 9$ & $81 \pm 5$ & $83 \pm 9$ \\
\hline \multicolumn{4}{|l|}{ Current medication } \\
\hline Diuretics & $62 \%$ & $69 \%$ & $45 \%$ \\
\hline ACE inhibitors & $50 \%$ & $25 \%$ & $45 \%$ \\
\hline$\beta$-blockers & $44 \%$ & $38 \%$ & $19 \%$ \\
\hline $\mathrm{CCl}$ & $12 \%$ & $0 \%$ & $0 \%$ \\
\hline
\end{tabular}

Abbreviations: ACE inhibitors, angiotensin conversor enzyme; $\mathrm{CCl}$, calcium channel inhibitor; F, female; M, male; PWV, pulse wave velocity; W/H, waist/hip.

$P>0.05$ for all variables between groups.

test as well as patients with non-cardiovascular functional limitations such as osteoarthritis. Subjects participating in regular physical activity (more than once a week) for the previous 6 months were also excluded.

This protocol was approved by the ethics committee of the study institution. All patients provided informed consent before participation.

\section{Study design}

This was a randomized, controlled trial to investigate the effects of continuous and interval exercise training on arterial stiffness and blood pressure in hypertensive subjects. First, all subjects underwent an exercise test to exclude electrocardiographic abnormalities. After 2 days, we performed PWV measurements by using a non-invasive device and 24 -h ambulatory blood pressure monitoring (ABPM). Referred patients with no exclusion criteria were randomized in a 2:2:1 ratio between the continuous, interval and control groups, respectively. A computer-generated randomization list was created by the statistician. The 2:2:1 randomization sequence was developed in blocks of five, including two continuous, two intervals and one control. The order of training and control within each block was also randomly assigned. Two days after the last session of the exercise training protocol (16 weeks), subjects were evaluated again for comparison, and the measurements were carried out by an investigator blinded to the exercise modality (Figure 1).

\section{Exercise test}

All subjects were asked to refrain from both strenuous physical activities and the consumption of any stimulants (for example, coffee, tobacco and alcohol) that might influence heart rate for $24 \mathrm{~h}$ before the exercise test and not to eat at least $2 \mathrm{~h}$ before the start of the test. They underwent exercise testing on a programmable treadmill (Quinton, Quinton, Seattle, WA, USA) with a standard 12-lead continuous electrocardiography monitor (TAB-SM 310), in a temperature-controlled room $\left(21-23^{\circ} \mathrm{C}\right)$ between 10:00 a.m. and 3:00 p.m. Blood pressure monitoring was performed by the auscultatory method. Resting heart rates were computed as the mean of the final $30 \mathrm{~s}$ of the resting period, and peak heart rates were the mean values of the final $30 \mathrm{~s}$ of effort before exhaustion. A satisfactory exercise test (modified Bruce protocol) was defined by symptoms of maximum effort. The maximum exercise heart rate predicted for age was calculated by subtracting the subject's age from 220 .

\section{Exercise training protocol}

All subjects performed the exercise training protocol in a room with temperature controlled at $20 \pm 1{ }^{\circ} \mathrm{C}$ for 16 weeks, three times per week (two supervised

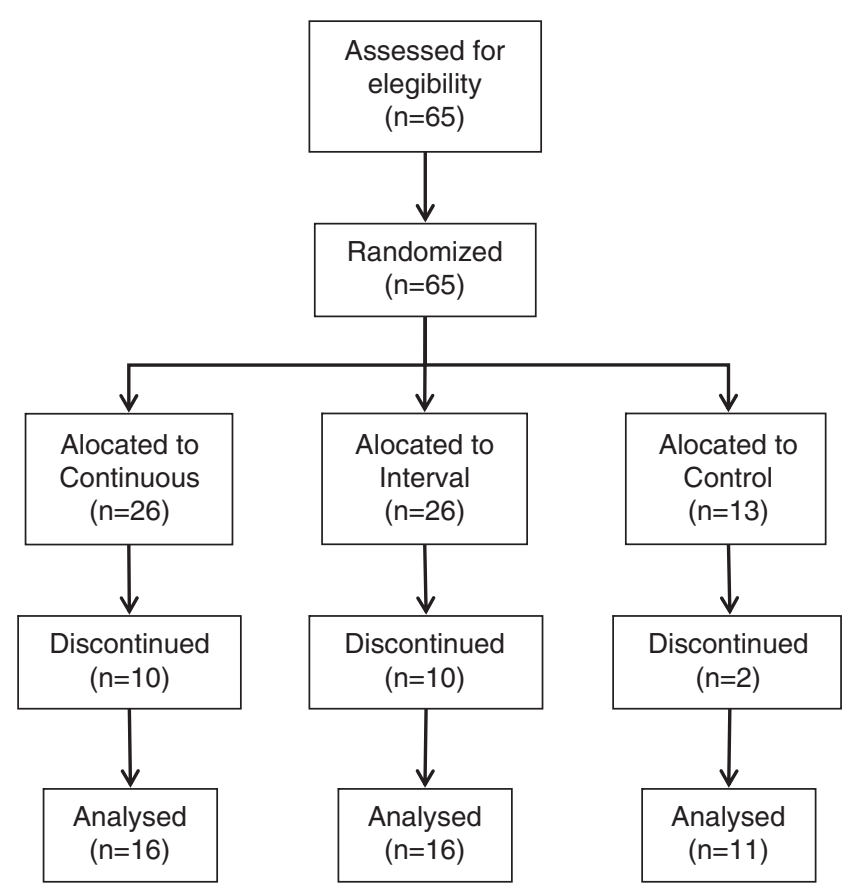

Figure 1 Study design.

and one non-supervised session). Supervised exercise sessions consisted of $10 \mathrm{~min}$ of warm-up stretching exercises, $40 \mathrm{~min}$ of exercise training on a treadmill (either continuous or interval exercises), $20 \mathrm{~min}$ of submaximal strength training and $10 \mathrm{~min}$ of cool-down exercises. During non-supervised exercise sessions the patients were instructed to follow the same protocol carried out during supervised sessions. The control group was advised to maintain their daily activities without exercise training during the 16-week period.

Exercise intensity was determined according to the workload reached during the maximal graded exercise test and was calculated to promote the same cardiovascular workload for both continuous and interval training. Continuous exercise was $40 \mathrm{~min}$ on a treadmill at $60 \%$ of reserve heart rate. Interval exercise was $40 \mathrm{~min}$ on a treadmill with intensity alternating between $50 \%$ ( $2 \mathrm{~min}$ ) and $80 \%(1 \mathrm{~min})$ of reserve heart rate, resulting in a mean workload of $60 \%$ $((50 \% \times 2)+80 \% / 3)$. Reserve heart rate was calculated as the difference between peak and resting heart rate, multiplied by the intensity of exercise and added to resting heart rate, according to the Karvonen method. Peak and resting heart rates were obtained during the graded exercise test. Each individual's heart rate was monitored during all exercise sessions with a heart rate monitor (Polar Beat, Polar Eletro, Kempele, Finland). Subjects were excluded if they did not perform more than $70 \%$ of the exercise sessions.

\section{Ambulatory blood pressure monitoring}

Twenty-four hour ABPM was performed on the non-dominant arm using an oscillometric device (SpaceLabs Redmond, Issaquah, WA, USA). The device was set to take readings every $15 \mathrm{~min}$ during the day and every $20 \mathrm{~min}$ during the night, and a new measurement was obtained after $2 \mathrm{~min}$ if the first one was unsuccessful. The software automatically edited values outside the normal physiological ranges and spurious readings because of external factors such as arm movement. Awake and asleep periods were determined by the patient before the examination. All subjects were instructed to maintain their usual activities and medications. The ABPM was set a day before each maximal cardiopulmonary exercise test. Data were accepted only if at least $75 \%$ of the measurements were successfully taken.

\section{Arterial stiffness}

Arterial stiffness was evaluated using carotid-femoral PWV measurements by a non-invasive automatic device (Complior; Colson, Garges les Gonesses, France) 
obtained by an experienced observer blinded to the patients' clinical characteristics and exercise modality. Briefly, common carotid artery and femoral artery pressure waveforms were recorded non-invasively by a pressure-sensitive transducer (TY-306-Fukuda; Fukuda; Tokyo, Japan). The distance between the recording sites $(D)$ was measured, and PWV was automatically calculated as $\mathrm{PWV}=D / t$, where $t$ represents pulse transit time. ${ }^{13}$ Measurements were repeated over 10 cardiac cycles, and the mean was used for the final analysis. Patients were told not to take anti-hypertensive medications on the day of the procedure. All of the tests were performed in the afternoon between 3 p.m. and 4 p.m. by the same physician who was blinded to the patient's group.

\section{Statistical analysis}

Descriptive analyses are presented as means with standard deviation. The blood pressure and PWV measurements were normally distributed. The two-way ANOVA (group $\times$ time) with repeated measures was used to compare the data. Bonferroni post hoc analysis was performed to evaluate the differences among groups. As systolic blood pressure has a direct influence on PWV measurements, we adjusted the values for the mean systolic blood pressure when comparisons among groups were done. Pearson's correlation was used to analyze associations between age and PWV decline in all groups.

Data were analyzed using the Statistical Package for Social Sciences for Windows, 11.5 (SPSS, Chicago, IL, USA). Statistical significance was defined as $P<0.05$.

\section{RESULTS}

The three groups had similar age, body mass index, office blood pressure, PWV values and current medication distribution (Table 1). Of the 65 randomized subjects, 43 completed the protocol. Seven subjects from the continuous group and seven from the interval group dropped out of the study for personal reasons. Two subjects were excluded because of changes in their medication (one in the continuous exercise and one in the control group) and five because they performed $<70 \%$ of the exercise sessions (two from the continuous group). One subject from the control group became pregnant (Figure 1). The protocol was well tolerated, and there were no differences in the training session between the continuous and interval groups ( $82 \pm 9$ and $81 \pm 10 \%$, respectively). Three women were postmenopausal (two in the interval group and one in the control group).

After 16 weeks of intervention, mean $24 \mathrm{~h}$, daytime and nighttime systolic and diastolic blood pressure did not change significantly in any of the three groups (Table 2). When we analyzed the two exercise groups together, exercise training significantly decreased the mean $24 \mathrm{~h}$ and daytime diastolic blood pressure, whereas diastolic blood pressure at nighttime and mean $24 \mathrm{~h}$, daytime and nighttime systolic blood pressure did not change (Figure 2). The decrease in blood pressure on ABPM was more significant in subjects whose office blood pressure

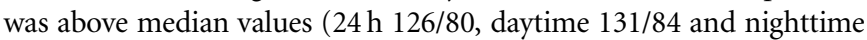
$117 / 73 \mathrm{~mm} \mathrm{Hg}$ ) at study inclusion (Figure 3 ).

Interval exercise training significantly decreased the carotid to femoral PWV values after 16 weeks, whereas no significant changes were observed in the continuous and control groups (Figure 4). The heart rate, systolic and diastolic blood pressure before and after 16 weeks, measured before PWV evaluation, did not differ among the three groups (Table 3). Moreover, there was no correlation between age and PWV decline for all groups (continuous $r=0.11$ and $P=0.34$, interval $r=0.30$ and $P=0.12$, and control $r=0.30$ and $P=0.18$ ).

Body mass index and waist circumference were unchanged after completion of the protocol in the three groups.

\section{DISCUSSION}

This is the first trial to evaluate the effects of interval exercise training on 24-h blood pressure and arterial stiffness in hypertensive subjects
Table 2 Ambulatory blood pressure monitoring pre- and post-16 week of intervention

\begin{tabular}{|c|c|c|c|c|c|c|}
\hline & \multicolumn{2}{|c|}{ Continuous } & \multicolumn{2}{|c|}{ Interval } & \multicolumn{2}{|c|}{ Control } \\
\hline & Pre & Post & Pre & Post & Pre & Post \\
\hline \multicolumn{7}{|l|}{$24 h$} \\
\hline SBP & $124 \pm 8$ & $124 \pm 9$ & $125 \pm 8$ & $123 \pm 9$ & $128 \pm 8$ & $127 \pm 9$ \\
\hline DBP & $80 \pm 9$ & $79 \pm 9$ & $80 \pm 5$ & $78 \pm 6$ & $83 \pm 8$ & $82 \pm 8$ \\
\hline \multicolumn{7}{|c|}{ Daytime } \\
\hline SBP & $129 \pm 10$ & $128 \pm 9$ & $128 \pm 9$ & $126 \pm 9$ & $131 \pm 9$ & $131 \pm 9$ \\
\hline DBP & $84 \pm 9$ & $82 \pm 9$ & $84 \pm 6$ & $81 \pm 6$ & $86 \pm 9$ & $86 \pm 9$ \\
\hline \multicolumn{7}{|c|}{ Nighttime } \\
\hline SBP & $113 \pm 7$ & $112 \pm 9$ & $116 \pm 9$ & $115 \pm 8$ & $119 \pm 10$ & $116 \pm 10$ \\
\hline DBP & $71 \pm 7$ & $70 \pm 8$ & $72 \pm 6$ & $71 \pm 5$ & $74 \pm 8$ & $73 \pm 8$ \\
\hline
\end{tabular}

Abbreviations: DBP, diastolic blood pressure in $\mathrm{mm} \mathrm{Hg}$; SBP, systolic blood pressure in $\mathrm{mm} \mathrm{Hg}$. No significant difference between groups prexpost intervention as analyzed by two-way ANOVA.

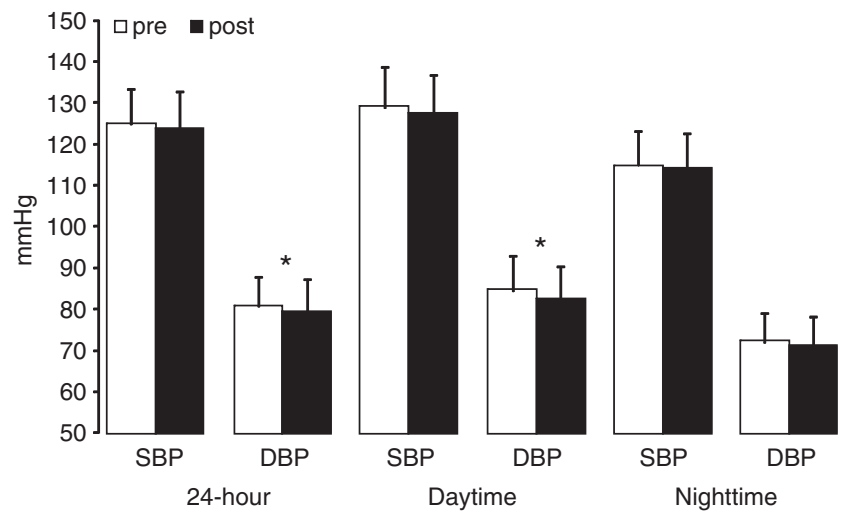

Figure 2 Average 24-h daytime and nighttime ABPM before and after 16 weeks of exercise training (continuous+interval groups). *Denotes $P<0.05$.

receiving long-term treatment. The main finding was the observation that exercise training, independent of the exercise training method, decreased blood pressure on ABPM. Moreover, only interval training improved arterial stiffness as evaluated by a decrease in carotidfemoral PWV.

\section{Blood pressure}

It is well known that aerobic exercise decreases blood pressure, mainly in the daytime. These blood pressure-lowering effects are more pronounced in hypertensive patients. ${ }^{14,15,16,17}$

One meta-analysis demonstrated that aerobic exercise decreases systolic and diastolic blood pressure by 6.9 and $4.9 \mathrm{~mm} \mathrm{Hg}$, respectively, in hypertensive patients vs. 2.4 and $1.6 \mathrm{~mm} \mathrm{Hg}$ in normotensive subjects. ${ }^{15}$ Another meta-analysis, which included ambulatory blood pressure measurements, demonstrated that aerobic exercise decreases systolic/diastolic blood pressure by $4.5 / 2.4 \mathrm{~mm} \mathrm{Hg}$ during the daytime and $1.7 / 0.7 \mathrm{~mm} \mathrm{Hg}$ during the nighttime. ${ }^{18}$ Our study is in agreement with these results; blood pressure in our subjects decreased during the daytime, mainly in those with higher blood pressure values.

A few studies have analyzed the effects of different intensities of exercise training on blood pressure. These studies showed that 


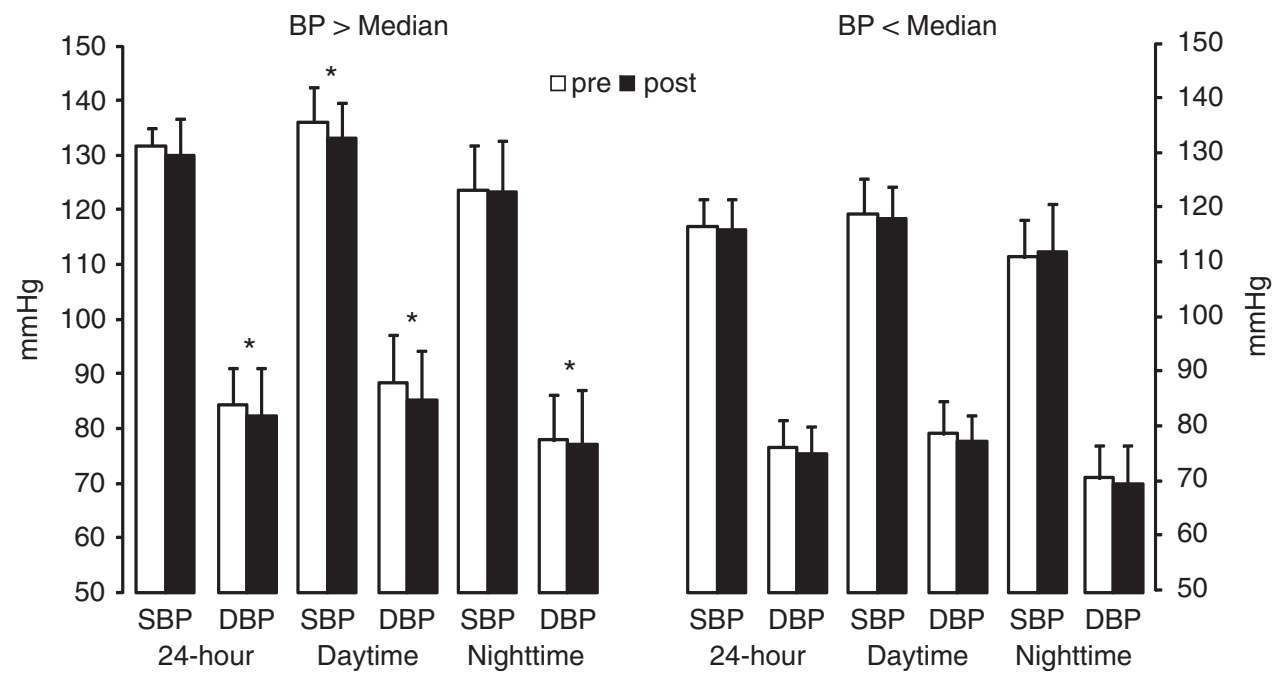

Figure 3 Average 24-h daytime and nighttime ABPM before and after 16 weeks of exercise training (continuous+interval groups) in the patients with control $\mathrm{BP}$ above and below the median value. ${ }^{*}$ Denotes $P<0.05$.

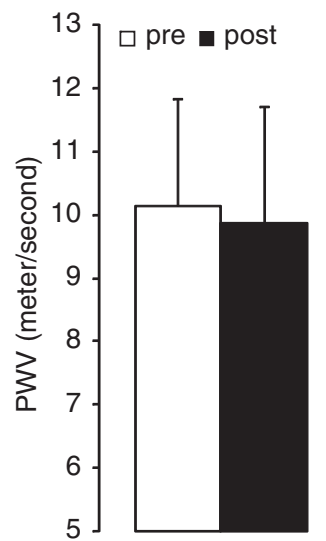

Continuous

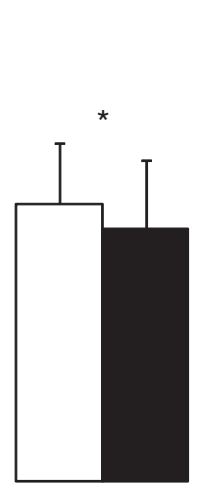

Interval

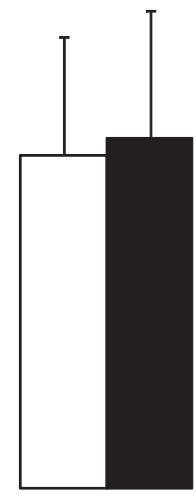

Control

Figure 4 PWV before and after 16 weeks of exercise training. *Denotes $P<0.05$.

Table 3 Blood pressure and heart rate levels before pulse wave velocity evaluation

\begin{tabular}{|c|c|c|c|}
\hline & Continuous & Interval & Control \\
\hline \multicolumn{4}{|c|}{ Systolic blood pressure ( $\mathrm{mm} \mathrm{Hg}$ ) } \\
\hline Pre & $136 \pm 10$ & $134 \pm 11$ & $134 \pm 13$ \\
\hline Post & $129 \pm 10$ & $128 \pm 10$ & $130 \pm 10$ \\
\hline \multicolumn{4}{|c|}{ Diastolic blood pressure $(\mathrm{mm} \mathrm{Hg})$} \\
\hline Pre & $89 \pm 9$ & $90 \pm 8$ & $89 \pm 11$ \\
\hline Post & $86 \pm 7$ & $86 \pm 7$ & $86 \pm 6$ \\
\hline \multicolumn{4}{|c|}{ Heart rate (b.p.m.) } \\
\hline Pre & $78 \pm 7$ & $80 \pm 11$ & $80 \pm 7$ \\
\hline Post & $76 \pm 9$ & $78 \pm 12$ & $79 \pm 7$ \\
\hline
\end{tabular}

No significant difference between groups prexpost follow-up as analyzed by two-way ANOVA.

exercise intensity does not influence the level of blood pressure decline. ${ }^{7,18}$ Our study is in agreement with those findings and shows that exercise intensity does not influence the decrease in blood pressure.

Several factors are involved in the blood pressure decline mediated by exercise training, but the improvement in peripheral vascular resistance seems to have the most important role. The decrease in sympathetic nervous system activity, ${ }^{19}$ effects on baroreflex control ${ }^{20}$ and improvement in nitric oxide production and action (endothelial function $)^{21}$ are probably involved in the anti-hypertensive effects of exercise. Moreover, effects on arterial remodeling, ${ }^{22}$ angiogenesis ${ }^{23}$ and arterial distensibility ${ }^{24}$ might be related to the blood pressure decline after aerobic exercise training.

\section{Arterial stiffness}

The aging process leads to a progressive increase in arterial stiffness, especially in large arteries, as has been clinically observed in elderly patients. Indeed, in hypertensive subjects this increase is more pronounced. ${ }^{25}$ Aerobic exercise training seems to attenuate arterial stiffness in healthy subjects, but the results in hypertensive patients are controversial. ${ }^{25}$ Twelve weeks of aerobic exercise training, at $70-75 \%$ of the maximum heart rate, increased the carotid compliance of healthy subjects to the same values as endurance-trained men. ${ }^{2}$ However, $8-12$ weeks of aerobic exercise training at $65-70 \%$ of the maximum heart rate did not improve arterial stiffness in patients with systolic hypertension. ${ }^{24,26}$ In our study, after 16 weeks of training, arterial stiffness, measured by PWV, decreased only in the aerobic interval training group. The exercise intensity and the severity of disease could explain these conflicting results.

The mechanisms involved in arterial stiffness are degradation of the elastic matrix, endothelial dysfunction, hypertrophy and hyperplasia of the smooth muscle cells and increase in collagen content. ${ }^{27,28}$ These mechanisms seem to be more accelerated in hypertensive subjects. ${ }^{28,27}$ On the other hand, an experimental study showed that the magnitude of wall distension promoted by a given shear stress affects the change in arterial stiffness through mechano-biochemical signaling that compromises vasorelaxation. ${ }^{29}$ Thus, aerobic exercise training with increased intensity or duration should be required to better improve arterial stiffness in hypertensive subjects. Nevertheless, in healthy subjects with less stiff arteries, a short duration and low intensity of exercise could be sufficient to improve arterial function. ${ }^{2,4}$ In this context, aerobic exercise with increased intensity could be required to improve arterial stiffness in hypertensive subjects, as observed in our study. As younger subjects may have less stiff arteries, and they may 
react with a greater PWV drop, one might argue that a 5-year difference between continuous and interval training may have influenced the results. However, this age difference was not statistically significant. Moreover, we did not find significant correlation between age and PWV drop.

\section{Clinical implications}

The reduction in blood pressure after both training protocols, although observed mainly in individuals with more elevated baseline levels, has important clinical implications because it has been documented that a reduction of only $2 \mathrm{~mm} \mathrm{Hg}$ in systolic blood pressure decreases mortality from cerebral vascular accidents by $10 \%$ and cardiovascular disease by $7 \% .^{30}$

Arterial stiffness has been shown to be an independent predictor of cardiovascular and all-cause mortality ${ }^{31}$ in hypertensive patients. Sedentary lifestyle and anti-hypertensive treatment resulted in an increase of $0.8 \mathrm{~m} \mathrm{~s}^{-1}$ of PWV in 6 years. ${ }^{27}$ Our study shows that only 16 weeks of interval training caused a drop in PWV of $0.41 \mathrm{~m} \mathrm{~s}^{-1}$ in a similar population.

Regular physical activity is an effective intervention with respect to these factors, decreasing the mortality rate for cardiovascular disease and all causes of diseases in hypertensive patients, ${ }^{32}$ attenuating the increase in the arterial stiffness associated with age, ${ }^{2}$ as well as reducing genetic susceptibility to this increase. ${ }^{33}$ In addition, physical activity helps to control the metabolic variables related to hypertension and arterial stiffness. ${ }^{34}$

\section{Study limitations}

This study has several limitations. The percentage of adherence to exercise training was $61 \%$ for both the interval and the continuous groups, which is a low level of adherence. There were two supervised training sessions per week; however, the patients were instructed to perform a non-supervised exercise session. Still, it has been shown that there is no significant difference in blood pressure reduction between an exercise training program requiring two sessions per week as compared with three sessions. ${ }^{35}$ In addition, we do not know whether our results would have been different for those whose hypertension was controlled compared with those with uncontrolled hypertension.

\section{Perspectives}

Interval and continuous exercise training were well tolerated in chronic hypertensive subjects treated with anti-hypertensive drugs. Blood pressure control improved with exercise training, but only interval exercise improved arterial stiffness, measured by PWV analysis, which re-affirms the need for the inclusion of physical activity in the treatment of hypertensive patients.

\section{CONFLICT OF INTEREST}

The authors declare no conflict of interest.

\section{ACKNOWLEDGEMENTS}

This study was supported by the Fundação de Amparo à Pesquisa do Estado de São Paulo, São Paulo-SP, Brazil (FAPESP \# 2002/06923-9). Guilherme V Guimarães (CNPq \# 304733/2008-3) was supported by Conselho Nacional de Pesquisa (CNPq).

1 National Heart Institute. Physical activity and cardiovascular health. JAMA 1996; 273 241-246.
2 Tanaka H, Dinenno FA, Monahan KD, Clevenger CM, DeSouza CA, Seals DR. Aging, habitual exercise, and dynamic arterial compliance. Circulation 2000; 102: 1270-1275.

3 Kakiyama T, Sugawa J, Murakami H, Maeda S, Kuno S, Matsuda M. Effects of shortterm endurance training on aortic distensibility in young males. Med Sci Sports Exerc 2005; 37: 267-271.

4 Cameron JD, Dart MD. Exercise training increase total systemic arterial compliance in humans. Am J Physiol 1994; 266: H693-H701.

5 Rowe JW. Clinical consequences of age-related impairments in vascular compliance. Am J Cardiol 1987; 60: 68G-71G.

6 Hodes RJ, Lakatta EG, McNeil CT. Another modifiable risk factor for cardiovascular disease? Some evidence points to arterial stiffness. J Am Geriatr Soc 1995; 43: 581-582.

7 American College of Sports Medicine. ACSM position on exercise and hypertension. Med Sci Sports Exerc 2004; 36: 533-553.

8 Fox El, Bartels RL, Billings CE, O'Brien R, Basan R, Mathews DK. Frequency and duration of interval training programs and changes in aerobic power. J Appl Physiol 1975; 38: 481-484.

9 Wenger HA, McNab RBI. Endurance training: the effects of intensity, total work, duration and initial fitness. J Sports Med 1975; 15: 199-203.

10 Rognmo O, Hetlandb E, Helgerudb J, Hoffb J, Slørdahla SA. High intensity aerobic interval exercise is superior to moderate intensity exercise for increasing aerobic capacity in patients with coronary artery disease. Eur J Cardiovasc Prev Rehabil 2004; 11: 216-222.

11 Wisløff U, Støylen A, Loennechen JP, Bruvold M, Rognmo Ø, Haram PM, Haram PM, Tjønna AE, Helgerud J, Slørdahl SA, Lee SJ, Videm V, Bye A, Smith GL, Najjar SM, Ellingsen $\varnothing$. Skjaerpe T. Superior cardiovascular,effect of aerobic interval training versus moderate continuous training in heart failure patients: a randomized study. Circulation 2007; 115: 3042-3044.

12 Ciolac EG, Guimarães GV, D Avila VM, Bortolotto LA, Doria EL, Bocchi EA. Acute effects of continuous and interval aerobic exercise on 24-h ambulatory blood pressure in longterm treated hypertensive patients. Int J Cardiol 2009; 17: 381-387.

13 Asmar R, Benetos A, Topouchian J, Laurent P, Pannier B, Brisac AM, Target R, Levy BI. Assessment of arterial distensibility by automatic pulse wave velocity measurement. Validation and clinical application studies. Hypertension 1995; 26: 485-490.

14 Pescatello LS, Kulikowich JM. The aftereffects of dynamic exercise on ambulatory blood pressure. Med Sci Sports Exerc 2001; 33: 1855-1861.

15 Cornelissen VA, Fagard RH. Effects of endurance training on blood pressure, blood pressure-regulating mechanisms, and cardiovascular risk factors. Hypertension 2005; 46: 667-675.

16 Ciolac EG, Guimarães GV, D'Avila VM, Bortolotto LA, Doria EL, Bocchi EA. Acute aerobic exercise reduces 24-h ambulatory blood pressure levels in long-term-treated hypertensive patients. Clinics 2008; 63: 753-758.

17 Carvalho VO, Ciolac EG, Guimarães GV, Bocchi EA. Effect of exercise training on 24hour ambulatory blood pressure monitoring in heart failure patients. Congest Heart Fail 2009; 15: 176-180.

18 Marceau M, Kouane N, Lacourciere Y, Cleroux J. Blood pressure: effects of different intensities on 24-h blood pressure in hypertensive subjects. Circulation 1993; 88: 2803-2811.

19 Wiegman DL, Harris PD, Joshua IG, Miller FN. Decreased vascular sensitivity to norepinephrine folowing exercise training. J App/ Physiol 1981; 51: 282-287.

20 Somers VK, Conway J, Johnston J, Sleight P. Effects of endurance training on baroreflex sensitivity and blood pressure in borderline hypertension. Lancet 1991; 337: 1363-1368.

21 Maeda S, Miyauchi T, Kakiyama T, Sugawara J, lemitsu M, Irukayama-Tomobbe Y, Murakami H, Kumagai Y, Kuno S, Matsuda M. Effects of exercise training of 8 weeks and detraining on plasma levels of endothelium-derived factors, endothelin-1 and nitric oxide, in healthy young humans. Life Sci 2001; 69: 1005-1016.

22 Dinenno FA, Tanaka H, Monahan KD, Clevenger CM, Eskurza I, DeSouza CA, Seals DR. Regular endurance exercise induces expansive arterial remodelling in the trained limbs of healthy men. J Physiol 2001; 534: 287-295.

23 Sexton $\mathrm{WL}$, Laughlin $\mathrm{MH}$. Influence of endurance exercise training on distribution of vascular adaptations in rat skeletal muscle. Am J Physiol 1994; 266: H483-H490.

24 Ferrier KE, Waddell TK, Gatzka CD, Cameron JD, Dart AM, Kingwell BA. Aerobic exercise training does not modify large-artery compliance in isolated systolic hypertension. Hypertension 2001; 38: 222-226.

25 Vaitkevicius PV, Fleg JL, Engel JH, O'Connor F, Wright JG, Lakatta LE. Effects of age and aerobic capacity on arterial stiffness in healthy adults. Circulation 1993; 88: 1456-1462.

26 Seals DR, Tanaka $\mathrm{H}$, Clevenger CM, Monahan KD, Reiling MJ, Hiatt WR, Davy KP, DeSouza CA. Blood pressure reductions with exercise and sodium restriction in postmenopausal women with elevated systolic pressure: role of arterial stiffness. J Am Coll Cardiol 2001; 38: 506-513.

27 Zieman SJ, Melenovsky V, Kass DA. Mechanism, pathophysiology, and therapy of arterial stiffness. Artherioscler Thromb Vasc Biol 2005; 25: 932-943.

28 Benetos A, Adamopoulos C, Bureau JM, Temmar M, Labat C, Bean K, Thomas F, Pannier B, Asmar R. Determinants of accelerated progression of arterial stiffness in normotensive subjects and in treated hypertensive subjects over a 6-Year period. Circulation 2002; 105: 1202-1207.

29 Peng X, Haldar S, Deshpande S, Irani K, Kass DA. Wall stiffness suppresses Akt/eNOS and cytoprotection in pulse-perfused endothelium. Hypertension 2003; 41: 378-381. 
30 Prospective Studies Collaboration. Age-specific relevance of usual blood pressure to vascular mortality: a meta-analysis of individual data for one million adults in 61 prospective studies. Lancet 2002; 360: 1903-1913.

31 Laurent S, Boutouyrie P, Asmar R, Gautier I, Laloux B, Guize L, Ducimetiere P, Benetos A. Aortic stiffness is an independent predictor of all-cause and cardiovascular mortality in hypertensive patients. Hypertension 2001; 37: 1236-1241.

32 Evenson KR, Stevens J, Thomas R, Cai J. Effect of cardiorespiratory fitness on mortality among hypertensive and normotensive women and men. Epidemiology 2004; 15: 565-572.
33 Greenfield JR, Samaras K, Campbell LV, Jenkins AB, Kelly PJ, Spector TD, Hayward CS. Physical activity reduces genetic susceptibility to increase central systolic pressure augmentation: a study of females twins. J Am Coll Cardiol 2003; 102: $1270-1275$.

34 Ciolac EG, Guimarães GV. Exercício físico e síndrome metabólica. Rev Bras Med Esporte 2004; 10: 319-324.

35 Takata KI, Ohta T, Tanaka H. How much exercise is required to reduce blood pressure in essential hypertensives: a dose-response study. Am J Hypertens 2003; 16: 629-633. 